\title{
Loop Antennas for Near-Field Multipolar-Expansion Identification: First Experimental Validations
}

\author{
Benjamin Vincent, Olivier Chadebec, Jean-Luc Schanen, Senior Member, IEEE, and Kévin Berger
}

\begin{abstract}
In this paper, electromagnetic compatibility is assessed using novel magnetic induction sensors based on the standard CISPR16-1 coils. The new coil shape allows the sensors to be sensitive only to specific components of the multipolar expansion, which is similar to a spatial filtering. The aim is to simplify the geometry of sensor coils introduced in the previous work by rotating the spherical-harmonic functions. This paper describes the mathematical tools required as well as the design method and concludes with the first experimental studies.
\end{abstract}

Index Terms-Electromagnetic compatibility, magnetic-field measurement, multipolar expansion, power electronics, transducers.

\section{INTRODUCTION}

$\mathbf{I}$ N ELECTROMAGNETIC compatibility studies, a compact model of the radiated magnetic field generated by powerelectronic systems enables the prediction of the field surrounding the device. This model can sometimes be obtained using numerical methods, but in most cases, the systems and associated phenomena are too complex to be numerically modeled. Another approach uses measurements of the radiated magnetic field close to the device in order to identify its equivalent magnetic model and then extrapolate the field in the surrounding space. In this paper, we focus on a multipolar-expansion model of the radiated magnetic field. This model of decomposition is well known for its ability to represent magnetic sources as a combination of known standardized sources (dipole, quadrupole, octupole, ...) [1].

It must be stressed that inaccuracies in measurement can strongly influence the determination of each term of the spherical-harmonic development. Several solutions can reduce these effects. In the case of punctual measurements, Keradec et al. [2] propose multiple measurement locations. In this work, the magnetic-field sensor is a small coil moving around the

Manuscript received July 31, 2009; revised April 26, 2010; accepted April 28, 2010. This work was supported in part by the competitiveness cluster Mov'eo and in part by the System@ @ic (O2M Project). Portions of this paper are reprinted or reused, by permission, from the Proceedings of the 4th International Conference on Electromagnetic Near-Field Characterization and Imaging (ICONIC 2009), Copyright 2009. The Associate Editor coordinating the review process for this paper was Dr. Jean-Fu Kiang.

B. Vincent, O. Chadebec, and J.-L. Schanen are with the Grenoble Electrical Engineering Laboratory (UMR CNRS 5269), Université de Grenoble (Grenoble INP-Université Joseph Fourier), 38402 Saint Martin d 'Hères, France (e-mail: benjamin.vincent@g2elab.grenoble-inp.fr; chadebec@g2elab. grenoble-inp.fr; schanen@g2elab.grenoble-inp.fr).

$\mathrm{K}$. Berger is with Ampère Laboratory (CNRS UMR5005), Université de Lyon, École Centrale de Lyon, 69134 Ecully Cedex, France.

Color versions of one or more of the figures in this paper are available online at http://ieeexplore.ieee.org.

Digital Object Identifier 10.1109/TIM.2010.2063052

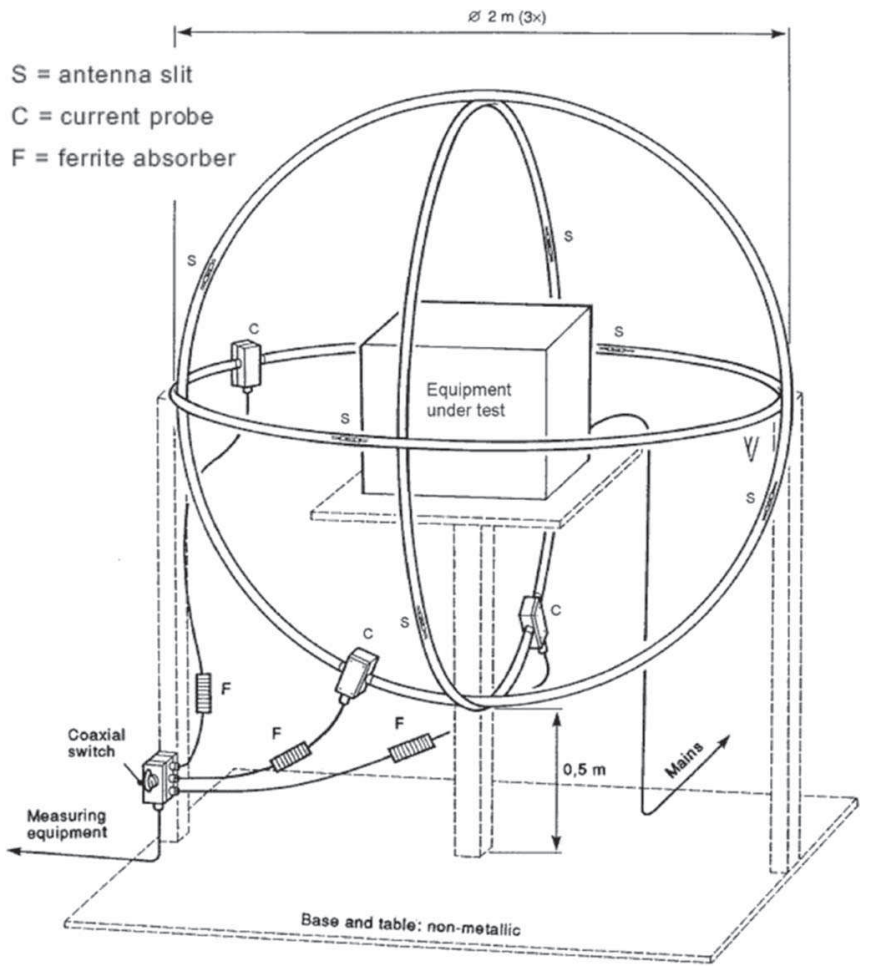

Fig. 1. From standard CISPR 16-1, the loop-antenna system, consisting of three mutually perpendicular large loop antennas.

object of study in order to measure the field at many points (up to 200). However, this measurement setup is complicated to build and can lead to inaccuracies in the positioning of the probe. An alternative is offered by Rouve et al. [3], who employ the punctual measurement but with an optimized number and positioning of fixed sensors to increase robustness of identification.

Another strategy consists in measuring the magnetic flux induced in large coils placed around the device in order to achieve a spatial integration and thus reduce positioning inaccuracies [4]. For example, to measure the first order of the decomposition (dipole) [5], the standard CISPR 16-1 [6] uses three orthogonal loops (Fig. 1), one for each dipole. The use of three identical coils with simple geometry facilitates the building of the sensor. Nevertheless, to ensure an accurate identification, the coils have to be large (at least $2 \mathrm{~m}$ in diameter). The resulting sensor is bulky and distant from the object of study. The magnetic field is weak, making it difficult to discriminate from electromagnetic noise. Although the sensor's measurement frequency range is limited, this standard applies for the medium frequency range: $9 \mathrm{kHz}$ to $30 \mathrm{MHz}$ [7]. 
In this paper, two improvements to this device are proposed: the identification of the quadrupolar terms (while maintaining identification of the dipolar terms) and the reduction of the sensor size. The measurement principle is similar to a spatial filtering: According to the coil shape, each sensor is sensitive to one specific component of the multipolar expansion. In a previous paper, we propose a set of coils that identify the first two orders ( $n=1$ and 2 , i.e., dipolar and quadrupolar terms) but are not sensitive to the following two orders (3 and 4). This results in a sensor with eight flux coils located on a measurement sphere (radius $r_{M}$ ). While the possibility of this new set of sensors has already been presented [8], we show here that this solution remains theoretical due to an infeasible measurement process. In this paper, we propose another approach. While only a few coils with a simple geometry are considered, the identification is performed by rotating these coils around the device. The measured fluxes in different positions are combined, and the equivalent multipolar terms can be determined due to a simpler measurement process. Here, this new solution is proposed and validated, and a pilot experiment is presented.

\section{Complete Coil Set for MultipolaR-DECOMPOSITION IDENTIFICATION}

\section{A. Assumptions for the Electromagnetic Field}

Power-electronic devices present low clock/commutation frequencies in comparison with standard electronic devices (about tens of kilohertz). Keeping in mind that the wavelength $\lambda$ of $100 \mathrm{kHz}$ in air is $3000 \mathrm{~m}$, the near-field approximation, defined by $r<\lambda / 2 \pi$ ( $r$ being the distance between the device and the point where the field is expressed), can be used near and below $100 \mathrm{kHz}$. In the identification and extrapolation of the components in our application, we can assume that the electrical and the magnetic fields are quasi-static, i.e., there is no delay between sources and electromagnetic effects. In other words, in the selected frequency range, propagation effects can be neglected. Moreover, a radiated electromagnetic field is created by both electric and magnetic sources [9]. However, for lowfrequency applications, it is commonly assumed that magnetic effects are dominant, and therefore, the electrical sources can be neglected. The radiated electromagnetic field is mainly created by current loops flowing inside the device. With a maximum frequency of $30 \mathrm{MHz}$, the quasi-static approximation is valid for up to $1.7 \mathrm{~m}$ away from the tested device.

\section{B. Magnetic Multipolar Expansion}

Multipolar expansion is a common tool used for electromagnetic-field representation [1]. Outside a sphere (radius $r_{V}$ ) that includes all radiation sources, the magnetic field can be fully described by its magnetic scalar potential $\psi$ solution of the Laplace equation [9]. For each frequency, we have

$$
\Psi(r, \theta, \varphi, f)=\frac{\mu_{0}}{4 \pi} \sum_{n=1}^{+\infty} \sum_{m=-n}^{+n} A_{n m}(f) \cdot \frac{1}{r^{n+1}} \cdot S_{n m}(\theta, \varphi)
$$

where $S_{n m}$ is the real harmonic spherical function of order $n$ and degree $m$, and $(r, \theta, \varphi)$ are the spherical coordinates whose origin is the center of the expansion. $A_{n m}(f)$ are the frequencydependent unknowns to be measured. Applying the gradient operator to (1), we get the multipolar expansion of the magnetic field for a near-field area

$$
\begin{aligned}
\mathbf{B}(r, \theta, \varphi, f) & =-\operatorname{grad}(\Psi(r, \theta, \varphi, f)) \\
\mathbf{B}_{n m} & =-\frac{\mu_{0}}{4 \pi} A_{n m}(f) \cdot \operatorname{grad}\left(\frac{1}{r^{n+1}} \cdot S_{n m}(\theta, \varphi)\right) .
\end{aligned}
$$

where the $S_{n m}$ spherical-harmonic functions are defined by

$$
S_{n m}(\theta, \varphi)=\Theta_{n m}(\theta) . \Phi_{m}(\varphi)
$$

with

$$
\begin{gathered}
\Theta_{n m}(\theta)=(-1)^{|m|} \sqrt{\frac{(n-|m|) !}{(n+|m|) !}} P_{n|m|}(\cos \theta) \\
\Phi_{m}(\varphi)= \begin{cases}\sqrt{2} \cos (m \varphi), & \text { if } m>0 \\
1, & \text { if } m=0 \\
\sqrt{2} \sin (|m| \varphi), & \text { if } m<0\end{cases}
\end{gathered}
$$

where $P_{n m}$ are the associated Legendre polynomials.

This development is hierarchical; for elements of the $n$th order, the field decreases by $1 / r^{n+2}$. A decomposition limited to the second order is sufficiently precise for normal powerelectronic structures, beyond the distance of extrapolation. Indeed, the higher orders rapidly become negligible. Therefore, there are eight coefficients to be determined (three for the first order, i.e., the dipolar terms, and five for second order, i.e., the quadrupolar terms).

\section{Initial Proposal for Component Identification}

As mentioned before, the measurement principle is similar to spatial filtering: According to the coil shape, each sensor is sensitive to one specific component of the multipolar expansion. The basic principle is to measure the magnetic flux through different coils in short-circuit mode on the measurement sphere $S_{M}$ (radius $r_{M}$ ) around the device. According to Lenz's law, the current in each coil will be directly proportional to the flux, and it is possible to calculate the flux (sum or difference) by measuring positive or negative values of the current.

In this approach, a set of coils is dedicated to only one $\mathbf{B}_{n m}$ component and not to the others. The flux across the surface delimited by the set of coils is expressed by

$$
\begin{aligned}
\operatorname{Flux}_{n m} & =\iint_{\text {Coil surface }}\left(\mathbf{B}_{n m}\left(r_{M}, \theta, \varphi\right) \cdot \mathbf{n}\right) d s \\
& =\frac{\mu_{0}}{4 \pi} \frac{(n+1)}{r_{M}^{n+2}} A_{n m} \iint_{S_{M}} S_{n m}(\theta, \varphi) d S
\end{aligned}
$$

where $\mathbf{n}$ is the unit outgoing normal of $S_{M}$ and Flux $n m$ corresponds to the sum of fluxes through the sensor coils. 


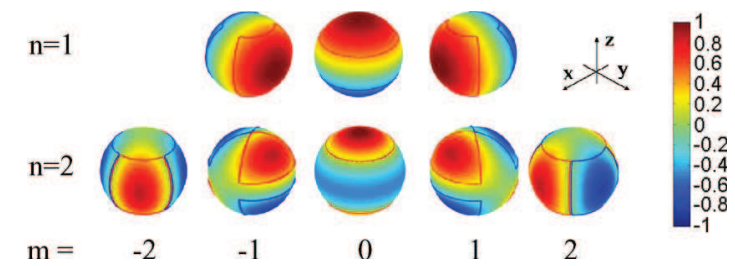

Fig. 2. Eight coil sensors for the identification of first- and second-order components. The flux is counted as positive for the red coils and negative for the blue ones. Functions $S_{n m}$ are presented using projection on the measurement sphere.

Equation (7) shows that such a coil configuration will enable the direct determination of the $A_{n m}$ coefficient, which is more robust than any indirect calculation. Moreover, we can see that the field distribution on a sphere $S_{M}$ is directly linked to the characteristics of the functions $S_{n m}$. Their study is therefore sufficient for determining the shape and configuration of the set of coils.

Using the proposed method, it is not possible to identify one component while filtering an infinite number of parasitic terms of the multipolar expansion. Thus, we consider only the first four orders of this expansion. The higher orders (above the fifth order) are considered negligible. Indeed, their influence decreases by $1 / r^{7}$, and the coils are located at two times $r_{V}$. Also, these types of power-electronic sources do not normally contain higher order components.

Using the initial system presented by Bergervoet and Van Veen [5], simple algebra shows that this kind of device dedicated to the determination of dipolar terms is influenced by octupolar components $(n=3)$ that decrease by $1 / r^{5}$. This destabilizing component can lead to measurement inaccuracies. In fact, if a large device is tested, the flux would be created by the tracked dipole and this additional term. The solution proposed by Bergervoet and Van Veen [5] is simply to increase the size of the loop (or the sphere) up to $2 \mathrm{~m}$ in diameter in order to reduce the octupolar contribution. However, this set of loops becomes very sensitive to external magnetic fields since the measurement surface area is so high.

In a new approach described in a previous paper [8], we proposed a system of coils to identify the first and second orders only. The theory was based on a careful study of the mathematical properties of spherical-harmonic functions. The resulting sensor had eight flux coils on a measurement sphere. To simplify the coil-design process, the shape of the sensor coils would have constant $\theta$ or $\varphi$ angles, i.e., these coils would follow only the meridians or parallels of the measurement sphere. This design constraint is suggested by (4), where the sphericalharmonic function $S_{n m}$ depends on the $\theta$ variable and on the $\varphi$ variable. These different sensor shapes are shown in Fig. 2 . The proposed solution lead to a much more compact system associated with a theoretical improvement in accuracy. Moreover, with the size of the loop being reduced, the propagation effect would not disturb the measurement. The proposed system would exhibit a higher frequency range than the large coils used by Bergervoet and Van Veen [5].

Unfortunately, this measurement setup was difficult to build. The coils were not located on the plane surfaces and were too numerous to construct. Even for a simple dipole identification,

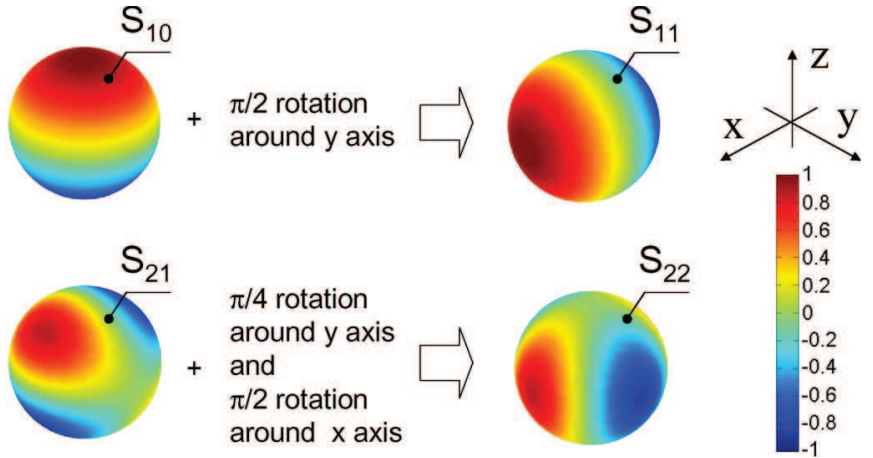

Fig. 3. Examples of rotation properties of spherical-harmonic functions.

the solution proposed for $n=1$ remained quite difficult. A simplification of this theoretical setup was necessary.

\section{New Approach Based on Rotating Sensors}

\section{A. Basic Idea}

The simplification of the measurement process has been presented in [10]. The aim was to simplify the previous solution (presented in [8]) in order to build a practical sensor. The basic idea comes from the properties of spherical-harmonic functions. With the rotations shown in Fig. 3, it is possible to generate all components of the same order $n$ with only one component. The first-order rotation dependence is obvious. For the $n=1$ case (Fig. 2), even if the associated sphericalharmonic functions are very similar, the sensor shapes obtained present markedly different geometries, although, obviously, the dipole basis must have symmetrical behavior along the $x-, y$, and $z$-axes. A solution with a single sensor shape associated with two rotations can lead to exactly the same identification. This solution has already been mentioned by Pasmooij [11], where a new shape could determine equivalent dipole terms. It corresponds to our $S_{10}$ sensors (equivalent to Helmholtz coils) associated with the two $\pi / 2$ rotations. This sensor presents an improvement over the system by Bergervoet and Van Veen [5] by ensuring the spatial filtering of the $n=3$ component.

The basic idea is that it is possible to generalize this property to design all sensors. In Fig. 2, two sensors seem particularly easy to build: the coils of the $A_{10}$ and $A_{20}$ sensors. Each of them is composed of two circular coils. In the following paragraphs, we will focus on these simple shapes.

\section{B. Example of $A_{21}$ Sensor Design}

The $A_{21}$ sensor is associated with the complex coil topology shown in Fig. 2. Using the ideas described earlier, it is possible to summarize the fluxes measured by the $A_{20}$ sensor oriented along different geometric axes to get the equivalent fluxes measured by the $A_{21}$ sensor. The geometrical rotation properties are detailed in [12]. The orthogonal properties of the spherical-harmonic base show that, if one component sensor is rotated, only components of the same order $n$ could create a parasitic flux through the coils. Therefore, the system of loops for detecting the $A_{21}$ component maintains the filtering capability for the other orders. The algebra is quite complicated 


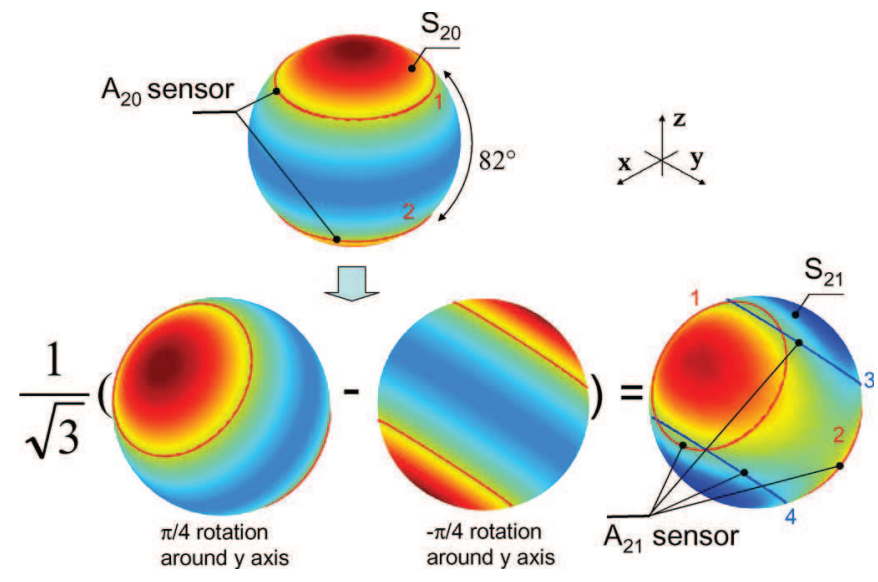

Fig. 4. Identification of $A_{21}$ parameter using $A_{20}$ sensor topology and associated rotation and flux combinations.

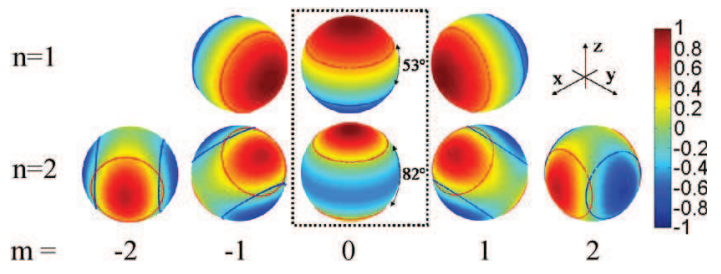

Fig. 5. Four simple circular loops associated with rotation to identify all the $n=1$ and $n=2$ components.

and will not be developed in this paper, but an illustration of this example is shown in Fig. 4. The $S_{21}$ function is generated from the rotation and composition of $S_{20}$

$$
S_{21}=\frac{1}{\sqrt{3}}\left(S_{20}^{\pi / 4}-S_{20}^{-\pi / 4}\right)
$$

where $S_{20}^{\pi / 4}$ represents the $S_{20}$ function rotated by an angle of $\pi / 4$ around the $y$-axis. The $A_{21}$ sensor is made by using the $A_{20}$ sensors associated with two rotations and a composition, as shown by (8).

\section{Final Topology}

Finally, we present here the simplification of the shapes obtained (Fig. 5). There are only four circular loops (two for the dipole identification and two for the quadrupolar one). This new set of loops provides an accurate identification of $A_{n m}$, like the first sensor shown in Fig. 2. The relationship between measured fluxes and $A_{n m}$ components is given by

$$
\left\{\begin{array}{l}
A_{1 m}=\frac{10^{8} r_{M}}{32 \pi} \operatorname{Flux}_{1 m} \\
A_{20}=\frac{6125 \cdot 10^{4} r_{M}^{2}}{3 \pi \sqrt{21}} \operatorname{Flux}_{20} \\
A_{2 m}=\frac{6125 \cdot 10^{4} \cdot r_{M}^{2}}{3 \pi \sqrt{21}}\left(\frac{1}{\sqrt{3}}\right) \operatorname{Flux}_{2 m}, \quad \text { for } m \neq 0
\end{array}\right.
$$

where Flux ${ }_{n m}$ corresponds to the total flux through the sensor coils. For example, for the $A_{21}$ sensor, according to Fig. 4, we have

$$
\text { Flux }_{21}=\text { flux }_{1}+\text { flux }_{2}-\text { flux }_{3}-\text { flux }_{4}
$$

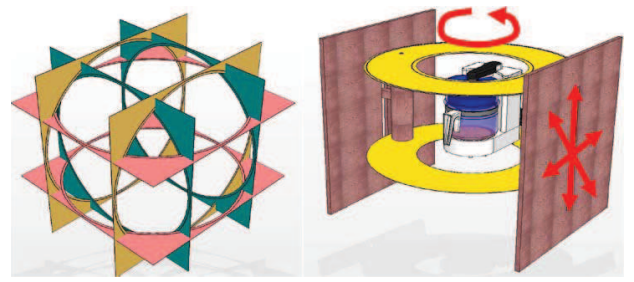

Fig. 6. Two suggestions for construction of the sensor. On the left is a static solution enabling the determination of the three dipole components. Construction is possible using three different radii of the measurement sphere. The construction on the right enables the identification of dipolar and quadrupolar terms (coils have three different axes, and device has two around the $z$-axis). The two $A_{10}$ and $A_{20}$ sensors have been located on the same plane to simplify the geometry, which changes the measurement radius.

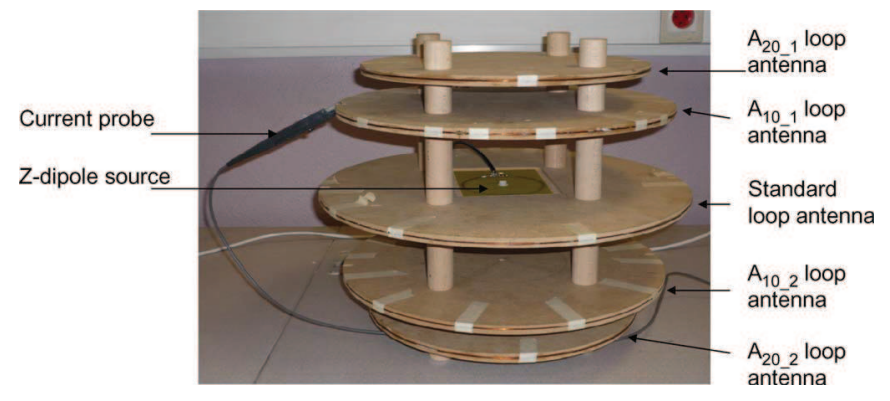

Fig. 7. Prototype photograph presenting the whole system of loop antennas (" $A_{10}$, " " $A_{20}$," and "standard").

where flux ${ }_{i}$ corresponds to the flux measured across the coil $i$ in Fig. 4.

In Fig. 5, the complexity of the system is significantly reduced for the coils, but it must be pointed out that the mechanical management of rotation can lead to new difficulties. Some ideas for their construction are shown in Fig. 6.

\section{Pilot Experiments}

In this pilot experiment, we built a prototype that includes only the two $A_{10}$ and $A_{20}$ sensors. They correspond to the $z$-components of the dipole and the quadrupole (black frame in Fig. 5). This paper will illustrate the improvement of the filtering capabilities and robustness over the standard system without addressing the construction problems due to the three axes. The prototype is shown in Fig. 7. All coils are designed around a measurement sphere of radius $r_{M}=0.225 \mathrm{~m}$.

\section{A. Prototype Presentation}

The first issue addressed when making the prototype was the choice of the field-measurement technique. There are two solutions: placing the coils in short-circuit mode and measuring the induced current or opening the coil and measuring the voltage. We choose the first alternative, allowing a nearly flat response in the frequency range $(9 \mathrm{kHz}-30 \mathrm{MHz})$ as explained by [13]. However, this solution leads to a new measurement difficulty. The mutual inductance between the coil sensors cannot be neglected, so the measurement must be achieved using one coil at a time, while the others remain in an open-circuit position. The current is measured using the usual current sensors (P6022, Tektronix) connected to a numerical oscilloscope. 


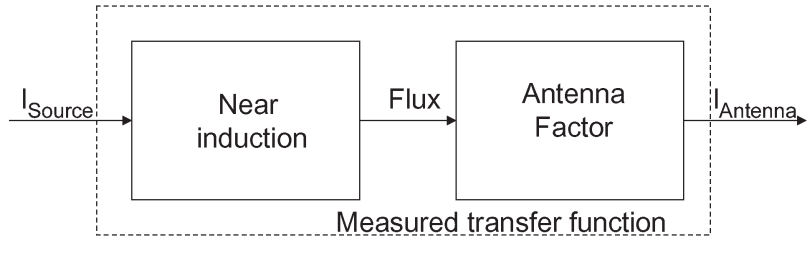

Fig. 8. Calibration process of each coil.
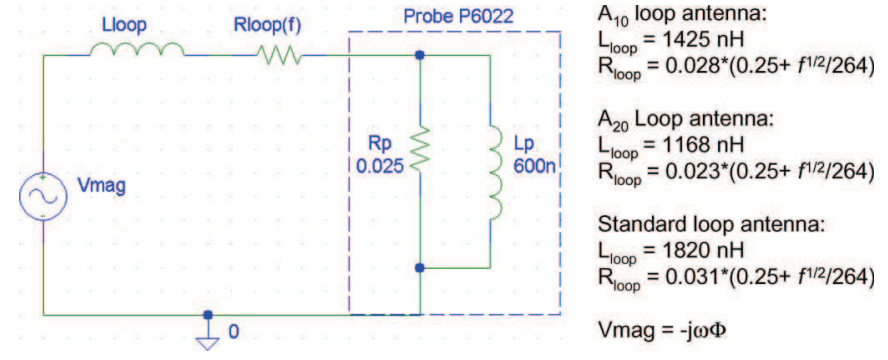

Fig. 9. Loop-antenna equivalent circuit.

In addition to the four-coil system, a simple loop antenna located in the middle plan has been added to demonstrate the robustness of this new approach. It corresponds to the initial $z$-dipole sensor proposed in [5].

With this configuration, the identified coefficients $A_{n m}$ are given using (9). For the standard sensor case with $z$-dipole source, we have, from (7)

$$
\begin{aligned}
& \text { Flux Standard } \\
& =\iint_{\text {Half }}\left(\mathbf{B}_{10}\left(r_{M}, \theta, \varphi\right) \cdot \mathbf{n}\right) d s \\
& =\frac{\mu_{0}}{4 \pi} \frac{(n+1)}{r_{M}^{n+2}} A_{10} \int_{\theta=0}^{\pi / 2} \int_{\varphi=0}^{2 \pi} S_{10}(\theta, \varphi) r_{M}^{2} \sin (\theta) d \theta d \varphi
\end{aligned}
$$

where $\mathbf{n}$ is the unit of outgoing normal of the measurement

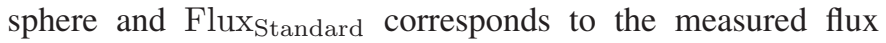
through the standard sensor coil. Therefore, after calculation, we get

$$
A_{10}=3.58 \cdot 10^{5} \text { Flux } \text { Standard }
$$

\section{B. Prototype Calibration}

The relationship between the flux and the measured current is needed to obtain the $A_{n m}$ coefficients (equivalent to the antenna factor). The calibration process is shown in Fig. 8.

To determine the antenna factor, two approaches are proposed. The first one consists in modeling the antenna factor using an $R L$ equivalent circuit, with the component being calculated analytically, shown in Fig. 9. The $R_{\text {loop }}$ equation takes into account the skin effect in the conductor. As mentioned before in Section II-A, this simple magnetic modeling of the loop is sufficient to characterize its behavior.

The second approach is to introduce a well-characterized magnetic source into our device. We have chosen a circular current loop (the value of the dipole is given by the current

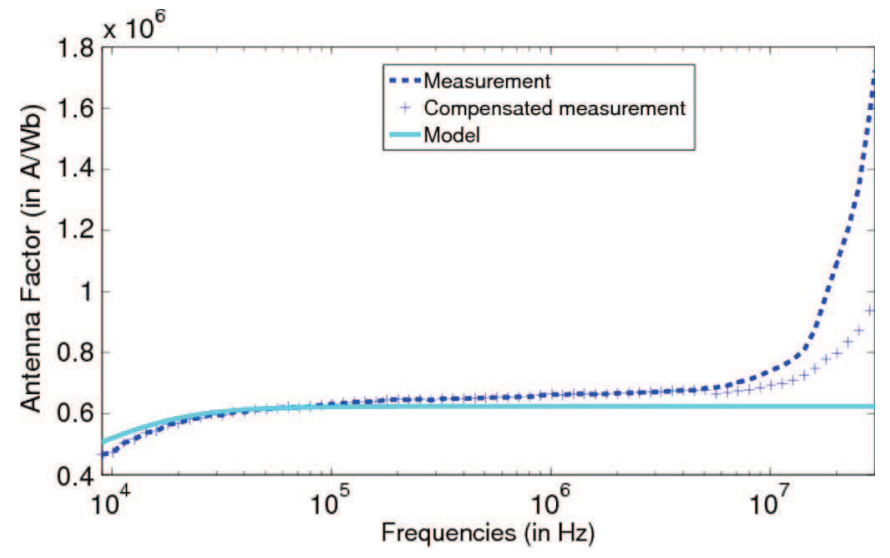

Fig. 10. $A_{10 \_1}$ loop-antenna factor from modeling circuit and calibration measurement.

TABLE I

IDENTIFICATION RESULTS WITH TWO DIFFERENT SOURCES Placed AT THE CENTER

\begin{tabular}{|c|c|c|c|c|}
\cline { 2 - 5 } \multicolumn{1}{c|}{} & \multicolumn{2}{c|}{$z$-dipole source $\left(\mathrm{mA} . \mathrm{m}^{2}\right)$} & \multicolumn{2}{c|}{} \\
\cline { 2 - 5 } \multicolumn{1}{c|}{} & Calculated & Measured & Calculated & Measured \\
\hline$A_{10}$ sensor & 7.854 & 7.955 & $/$ & -0.025 \\
\hline Standard sensor & 7.854 & 7.854 & $/$ & 0.030 \\
\hline$A_{20}$ sensor & $/$ & $9.2 \cdot 10^{-4}$ & 0.732 & 0.753 \\
\hline
\end{tabular}

multiplied by its surface area). The calibration process must be performed for each coil. The comparison between both approaches is shown in Fig. 10. They are highly consistent in the range from $10 \mathrm{kHz}$ to $10 \mathrm{MHz}$. The difference in high frequencies comes from interferences between the external strong magnetic field and the current measurement through the probe shield. In this case, a good solution would be to make a differential measurement, according to the instruction manual of the probe P6022. In Fig. 10, the antenna factor is obtained using a clamped probe around the conductor of the loop antenna. The compensated antenna factor is obtained by subtracting the signal measured with a probe placed as close as possible to the first probe and without the conductor inside. Even if the interferences are fairly reduced, a difference in modeling behavior persists. The following section describes an experiment performed at $1 \mathrm{MHz}$ and with the measured antenna factor.

\section{Measurement Results}

1) Filtering Capabilities: The validation of the $z$-dipole and $z$-quadrupole source identifications is summarized in Table I. These sources are centered, and their effects on the three sensors are measured one after the other. In all cases, the consistent results validate the $A_{10}$ and $A_{20}$ theoretical values. Thus, when the source is composed of a $z$-dipole, the result from the $A_{20}$ sensor measurement is very small. As with the standard sensor, the proposed solution exhibits good filtering of the second order. This filtering capability is less significant for the $z$-quadrupole case. Indeed, current intensities are close to the sensitivity limits of the oscilloscope, so the accuracy is reduced. 


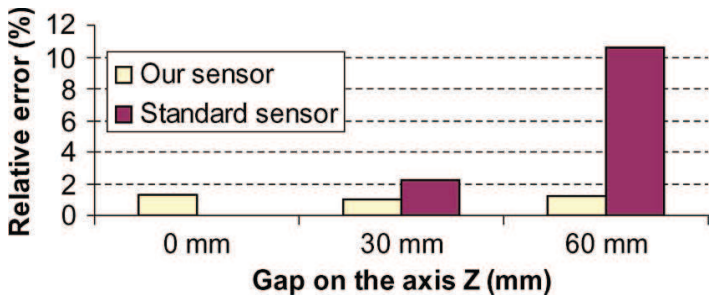

Fig. 11. Comparison of the relative error in identifications made by the standard sensor and our sensor, with respect to the theoretical $z$-dipole for different off-center placements of the source.

It should be noted in Table I that the result of the calibration using the $z$-dipole is fairly good, with only a $1.3 \%$ error.

The accuracy of the proposed sensor can be evaluated on the quadrupole identification results: less than $3 \%$ error, which is quite a good result.

However, the ideal case presented previously is far from likely source conditions, where the sources could be off-center and/or of a higher order than a pure dipole. This will be studied in the following section.

2) Robustness: The robustness of the proposed sensor is compared with the standard sensor by shifting the source loop away from the center of the sensors. Only the dipole identification is observed in this experiment. The measurement results in Fig. 11 show that an error occurs in the dipole determination with the standard sensor. However, despite the shifting of the source, the identification error remains the same with our sensor. With the standard sensor, the error increases up to $11 \%$. A decentered dipole implies a more complex field (i.e., higher orders in the decomposition). Since the filtering capability of the standard sensor is lower than our proposed sensor, the contribution of the higher orders to the measured signal corrupts the identification. The error is mainly attributed to the contribution of the third-order multipolar induction term in the standard sensor. The filtering capabilities of our sensors minimize this problem. The error is reasonable and lower than using the standard antenna since the latter is sensitive to thirdorder terms.

\section{CONCLUSION}

This paper has presented a new measurement system allowing the identification of an equivalent source for any radiating device. It provides the coefficients of the multipolar expansion, up to the quadrupole order. In comparison with previous solutions, the advantages are as follows.

1) Higher order determination, more robust behavior, and more compact system than the standard system using three simple loops.

2) Simple shapes used for construction, by rotating the simple sensors $A_{10}$ and $A_{20}$ to provide the other components. The complex shapes proposed in the previous work were efficient to filter the other orders but were too complicated to be built.

The new system has been detailed and validated using measurements on a single $z$-axis. The comparison with the standard sensor has shown increased robustness against shifts of a dipolar source, as expected.

Further work will focus on building the triple-axis system, improving the current measurement, and applying it to a complex radiating source, like a power-electronic converter.

\section{ACKNOWLEDGMENT}

The authors would like to thank C. Brun and F. Dumas for making the prototype, L.-L. Rouve for her clever advice about measurement, and D. Lyon for her linguistic assistance.

\section{REFERENCES}

[1] J. D. Jackson, Classical Electrodynamics, 3rd ed. New York: Wiley, 1998, pp. 407-456.

[2] J. P. Keradec, J. Lorange, J.-L. Schanen, B. Cogitore, T. Creuzet, and C. Brun, "Nano-teslameter for characterization of the fields emitted and received by wound electronic components," in Proc. 20th IEEE Instrum. Meas. Technol. Conf., May 2003, vol. 1, pp. 124-129.

[3] L.-L. Rouve, L. Schmerber, O. Chadebec, and A. Foggia, "Optimal magnetic sensor location for spherical harmonic identification applied to radiated electrical devices," IEEE Trans. Magn., vol. 42, no. 4, pp. 11671170, Apr. 2006.

[4] A. V. Kildishev, S. A. Volokhov, and J. D. Saltykov, "Measurement of the spacecraft main magnetic parameters," in Proc. IEEE Autotescon, 1997, pp. 669-675.

[5] J. R. Bergervoet and H. Van Veen, "A large-loop antenna for magnetic field measurements," in Proc. Zurich Int. Symp. EMC, 1989, pp. 29-34.

[6] Loop Antenna System for Magnetic Field Induced Current Measurements in the Frequency Range of $9 \mathrm{kHz}$, to $30 \mathrm{MHz}$, Standard CISPR 16-1, Annex P, pp. 230-237 and 396-409, 2002.

[7] M. Savi, T. Z. Gireli, F. A. Tirich, and C. A. F. Sartori, "Developing a Van Veen and Bergervoet antenna," in Proc. Int. Symp. Electromagn. Compat., 2004, vol. 2, pp. 717-720.

[8] B. Vincent, O. Chadebec, J.-L.Shanen, C. A. F. Sartori and L. Krahenbühl, "New robust coil sensors for near field characterization," in J. Microw. Optoelectron. Electromagn. Appl., vol. 8, no. 1, pp.64S-77S, Jun. 2009. [Online]. Available: http://www.sel.eesc.usp.br/jmo/

[9] C. J. Bouwcamp and H. B. G. Casimirc, "On multipole expansion in the theory of electromagnetic radiations," Physica, vol. 20, no. 1-6, pp. 539554, 1954.

[10] B. Vincent, O. Chadebec, J.-L. Schanen, and K. Berger, "Loop antennas for near field multipolar expansion identification: First experimental validations," in Proc. 4th Int. Conf. Electromagn. Near-Field Characterization Imaging (ICONIC), Taipei, Taiwan, Jun. 2009.

[11] W. A. Pasmooij, "A 'Helmholtz' large loop antenna system for improved magnetic field measurements," in Proc. EMC Conf., 1992, pp. 143-148.

[12] R. Green, "Spherical harmonic lighting: The gritty details," in Proc. Game Developers Conf., 2003, pp. 1-47.

[13] S. Schuh, H. Rossmanith, and M. Albach, "Reduction of magnetic field strength on PCB level laboratory for power electronics students," in Proc. Eur. Conf. Power Electron. Appl., Sep. 2-5, 2007, pp. 1-7.

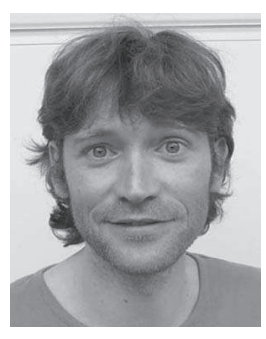

tronic systems.
Benjamin Vincent was born in 1974. He received the M.S. degree in robotics from Université Pierre et Marie Curie, Paris, France and from the University of Paris XI, Paris, in 2001. He is currently working toward the Ph.D. degree in Grenoble Electrical Engineering Laboratory (G2ELab), Université de Grenoble, Grenoble, France.

His research focuses on electromagnetic-field computation methods and measurements dedicated to inverse-problem theory. His applications deal with the electromagnetic compatibility of power elec- 


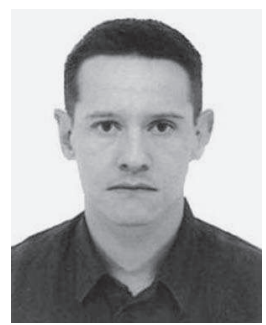

Olivier Chadebec was born in 1973. He received the Diploma in electrical engineering and the Ph.D. degrees from the Grenoble Institute of Technology, Grenoble, France, in 1997 and 2001, respectively.

$\mathrm{He}$ is currently a CNRS Research Associate with the team "Modeling and CAD in Electromagnetism," Grenoble Electrical Engineering Laboratory (G2ELab), Université de Grenoble, Grenoble. His current interests include electromagnetic-field computation methods, inverse-problem theory, and low-magnetic-field measurements.

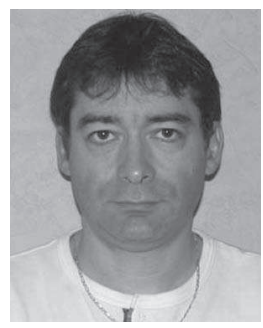

Jean-Luc Schanen (M'99-SM'04) was born in 1968. He received the Diploma in electrical engineering and the Ph.D. degrees from the Grenoble Institute of Technology, Grenoble, France, in 1990 and 1994, respectively.

He has been with the Grenoble Electrical Engineering Laboratory, St. Martin d'Hères, France, since 1994, working in the field of power electronics. He is currently a Professor with Grenoble Institute of Technology. His main activities concern the technological design of power converters. His research team uses (or develops) all kinds of modeling tools in order to improve the performance of power-electronic converters, including electromagnetic compatibility and thermal aspects.

Dr. Schanen is a senior member of the IEEE Power Electronics Society and IEEE Industry Applications Society and was Chairman of the Power Electronics Devices and Components Committee of the IEEE Industry Applications Society between 2006 and 2007 .

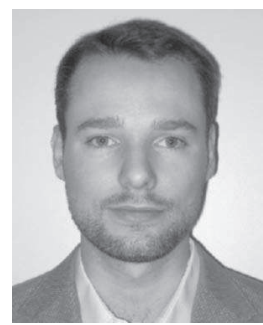

Kévin Berger was born in Montbéliard, France, on January 16, 1980. He received the Ph.D. degree in electrical engineering from Université Henri Poincaré, Nancy, France, in 2006.

$\mathrm{He}$ was with the Grenoble Electrical Engineering Laboratory (G2Elab) and the Néel Institute, Grenoble, France, in 2006 as a Postdoctoral Researcher in order to work on superconductor modeling using FLUX software and a conduction-cooled SMES. Since September 2008, he has been with the Ampère Laboratory, Université de Lyon, Ecully, France, working on radiated EMC models for power electronic devices. His research interests deal with the design and modeling of superconducting devices, electrical characterization of superconducting materials, near-field modeling, and measurements. 